\title{
TÚ Y USTED : DOS PRONOMBRES DE CORTESÍA EN EL ESPAÑOL ACTUAL. DATOS DE UNA COMUNIDAD PENINSULAR ${ }^{1}$
}

\author{
José LuIs Blas ARroyo \\ (Universidad Jaume I de Castellón)
}

\begin{abstract}
RESUMEN
The empiric data of this research, developed on a Spanish speech community show the social progression on the use of pronoun $t \hat{u}$, even in formal contexts reserved so far to the form usted. On the other hand, the paper considers the possibility of the dicotomic alternation between those pronouns of address in contemporary Spanish as an expression of two classes of politeness: positive politeness and negative politeness (Brown \& Levinson 1987). The aim of sociolinguistics and other related disciplines is to analyses the psychological, social and contextual factors that may move individuals and social groups to choose one model of address or another. Some speakers, like younger people or women, especially in non instrumental speech events and situations, have a significant correlation with the use of $t u ́$ in the speech community studied in the paper.
\end{abstract}

\section{Introducción.}

En el seno de la disciplina sociolingüística cada vez parecen más enfrentadas dos posiciones teóricas que analizan los fenómenos de la variación desde

1 La realización de este trabajo ha sido posible gracias a una beca concedida por la Fundación Caixa de Castellón en el marco anual del «Plan de Promoción de la Investigación: Convocatoria General de Ayudas para Proyectos de Investigación» (Año 1993). Agradecemos a esa institución financiera la ayuda económica prestada.

Aprovechamos también la ocasión para agradecer encarecidamente la ayuda prestada por Elena Arana Arbide a lo largo de la investigación, especialmente en el desarrollo del trabajo de campo. Sin ella, es muy posible que las páginas siguientes no hubieran sido escritas. 
ópticas notablemente diferentes. Por un lado, la que podríamos denominar corriente tradicional, de inspiración básicamente laboviana, aborda el origen y la naturaleza de dicha variación en el valor social de grupo que ciertas formas lingüísticas tienen asociado en una determinada comunidad de habla. En los últimos tiempos, sin embargo, y tras un periodo suficientemente amplio de revisión del trabajo realizado por el movimiento anterior, cada vez es mayor el número de investigadores que se decantan por una visión distinta - más cualitativa que cuantitativa- de las relaciones entre lengua y sociedad. Una visión que pone el énfasis en los mecanismos de la interacción verbal y en la que, por tanto, se consideran tanto o más importantes que la propia identidad social del hablante, determinados aspectos contextuales de la comunicación como el tipo de participantes, la clase de actividad social desarrollada mediante el lenguaje, el tono de la interacción, el grado en que los interlocutores participan de unas expectativas culturales comunes, las diferencias de poder y la distancia social entre ellos, etc. Desde hace unos años, a esta nueva fase de la investigación sociolinguíística, que tanto debe a disciplinas colaterales como la pragmática , la etnografía del habla o la etnometodología suele conocérsela con el nombre de sociolingüística interaccional (Kerbrat-Orechhini 1990: 9; Schiffrin 1994: 97).

Entre los temas en que esta variante de la sociolingüística ha puesto su atención últimamente queremos destacar en este trabajo el ámbito de los sistemas interpelativos, es decir, todas aquellas estructuras basadas en la combinación de elementos léxicos y gramaticales que los hablantes de una determinada lengua utilizan para apelar a sus semejantes (Dumitrescu 1975-76: 81, apud Cellard (1975)). Y en particular nos detendremos en los tratamientos, a juicio de algunos «the most conspicous intrusions of social factors into language structure» (Brown y Levinson 1987: 179). En opinión de estos dos autores, tales elementos representan: «(a) direct grammatical encodings of relative social status between participants, or between participants and persons or things referrered to in the communicative event», y por tanto, pueden ser consideradas perfectamente como integrantes del sistema deíctico de una lengua, en concreto como manifestaciones de la llamada deíxis social (vid también Fillmore 1975; Comrie 1976). Una clase de tratamientos bastante extendida entre las lenguas del mundo es la constituida por un sistema dual de pronombres, como las formas tú y usted del español, de las que nos ocuparemos en las páginas siguientes.

El problema de los tratamientos, cuyas consecuencias se dejan notar en numerosos ámbitos ${ }^{2}$, se halla íntimamente vinculado a la estructura social de cada sociedad. La bibliografía especializada ha subrayado, por ejemplo, la

2 Véase una opinión semejante en el artículo de Dumitrescu (1975-76: 81-86) donde se compara el sistema del español con el del rumano. 
importancia decisiva de esta clase de reglas de alternancia (Ervin-Tripp 1972: 93) para poder alcanzar una competencia comunicativa adecuada en lenguas que, como el español, poseen dobletes pronominales con implicaciones sociolingüísticas diferentes. A este respecto, Criado de Val (1972: 179) señala también: «en todo diálogo hay una mutua valoración (categoría social, grado de familiaridad, edad, etc) de los interlocutores. La expresión más directa de esta valoración por el lenguaje son los tratamientos, cuyo sistema varía grandemente de unos idiomas a otros y constituye a menudo una verdadera dificultad para su mutua traducción... Hay idiomas como el inglés y el latín, que apenas establecen diferencias de tratamiento, y otros, como el español y sobre todo el portugués, en los cuales es necesario un profundo conocimiento no sólo del idioma, sino de las costumbres sociales, para acertar con el tratamiento oportuno y justo». Un ejemplo extremo de la importancia social que adquiere el aprendizaje de estas normas en algunas comunidades humanas lo constituyen determinadas culturas orientales, como la japonesa o la coreana, donde tales sistemas han alcanzado un grado elevado de gramaticalización y complejidad social (Coulmas 1992: 303). Sin ir tan lejos, la propia historia española está plagada de ejemplos que demuestran la trascendencia social - y hasta penal, en ocasiones-- de estas cuestiones en una sociedad exacerbadamente puntillosa hasta tiempos bien recientes (Camargo 1972/73: 355; Lapesa 1970: 144-57; Alba de Diego y Sánchez Lobato 1980: 95).

Nos encontramos, en consecuencia, ante una de las zonas más inestables de la lengua, sujeta como probablemente ninguna otra a las modificaciones e irregularidades que impone el continuo devenir histórico y social y por tanto, uno de los terrenos más aptos para el estudio del cambio lingüístico en marcha. (Labov y otros 1968$)^{3}$. Ello explica probablemente por qué el trabajo pionero de Brown y Gilman (1960) - punto de partida indiscutible de gran parte de la investigación posterior sobre estos temas - tratara sobre la evolución de los sistemas pronominales de tratamiento ${ }^{4}$ en diversas lenguas europeas. En dicho estudio, se concluía, por ejemplo, que las principales reglas pragmáticas que han regulado el uso de tales formas desde la Edad Media hasta épocas contemporáneas han sido el poder y la solidaridad. Brown y Gilman (1960: 255) concebían el primero como el eje vertical de las relaciones sociales: «one person may be said to have power over another in the degree that he is able to control the behavior of the other». Por consiguiente, la presencia del poder en la

3 Un ejemplo de las posibilidades que este tema of rece desde el punto de vista de las variaciones que la propia evolución histórica impone al sistema pronominal es el estudio de Fontanella de Weinberg (1970) sobre las formas de tratamiento en el español bonaerense a lo largo de este siglo. A partir de la comparación de ciertos textos teatrales de principios de siglo entre sí y con respecto a otras obras actuales, la autora argentina llega a la conclusión de que ambos cortes en el tiempo revelan la existencia de cambios en vías de realización.

4 Como es ya habitual en esta clase de estudios, adoptamos la convención de utilizar las siglas $T / V$ para aludir a las formas de solidaridad y respeto respectivamente. 
interacción verbal desemboca necesariamente en la asimetría de trato: "power is a relationship between at least two persons, and it is nonreciprocal in the sense that both cannot have power in the same area of behavior». El corolario del poder en su aplicación al sistema pronominal es, por lo tanto, la elección de formas diferentes según la jerarquía relativa del interlocutor: el superior dirige $\mathrm{T}$ al inferior mientras recibe $\mathrm{V}$ de éste. En la sociedad actual suelen aducirse factores como la diferencia de estatus social, la autoridad o la distancia generacional como determinantes del trato asimétrico.

El eje horizontal de las relaciones corresponde, por el contrario, a la dimensión psicosocial de la solidaridad, entendida como el ámbito de las relaciones generales simétricas entre los individuos, lo que traducido al caso que nos ocupa significa el intercambio de $\mathrm{T}$ o $\mathrm{V}$ según el grado que dicho parámetro adquiere en la interacción, máximo en el caso de la primera forma y mínimo en la segunda. Variables sociales como las relaciones de parentesco, las afinidades de grupo - por sexos, edad, nacionalidad, aficiones, afiliaciones...- se dice que favorecen el intercambio recíproco en nuestros días (Alba de Diego y Sánchez Lobato 1980: 98$)^{5}$

La principal conclusión del trabajo de Brown y Gilman es que en las lenguas europeas dotadas de alternancias pronominales ha habido en los últimos siglos un cambio lento, pero progresivo, en favor de las normas asociadas con la solidaridad en detrimento de las vinculadas al poder, o lo que es lo mismo, un aumento de los tratamientos recíprocos entre los interlocutores. Dicho esquema general, que afecta a numerosas lenguas, a veces no relacionadas genéticamente, ha sido comprobado por lo general en diversos estudios sobre el español a ambos lados del Atlántico ${ }^{6}$. Una de las consecuencias más importantes de este fenómeno ha sido la progresiva extensión del tuteo recíproco en determinados ámbitos sociales para los que hasta hace poco estaba vedado, una evolución que se ha hecho más evidente en las últimas décadas en las que la democracia y el progreso social parecen haberse afianzado en diversas áreas del mundo hispánico, sustituyendo con ello a relaciones pretéritas generalmente mucho más jerarquizadas?

\footnotetext{
5 Para un desarrollo más pormenorizado de las normas de poder y solidaridad desde un punto de vista psicosocial puede acudirse al trabajo posterior de Brown (1965).

6 Sin pretensiones de exhaustividad, ofrecemos a continuación una relación de obras donde se ha abordado esta cuestión en relación con nuestra lengua: Alonso (1968); Eguiluz (1962); Fontanella (1970); Fontanella y Najt (1969); Fox (1970); Keller (1974); Gold (1974) Lapesa (1970); Lastra de Suárez (1972); Marín (1972); Criado de Val (1972; 1973); Dumitrescu (1975-76); Brown (1975); Miquel y Vergés (1963); Polo (1975); Solé (1970); Borrego, J. y otros (1978); Weinerman (1976); Aguado (1980); Ringer (1985); Ruiz Morales (1987).

7 Así ocurre, sin ir más lejos, en el ámbito de las relaciones familiares en las que hasta hace poco el tratamiento asimétrico entre generaciones diferentes era una norma bastante generalizada, especialmente en áreas rurales y entre las capas sociales más bajas (véase, entre otros, Solé 1970; Fontanella 1970b; Lastra de Suárez 1972; Keller 1974; Borrego y otros 1978).
} 


\section{Los pronombres de tratamiento y la cortesía.}

En el seno de la sociolingüística interaccional el análisis de los pronombres de tratamiento se ha insertado últimamente en el contexto de los estudios sobre la cortesía, uno de los universales pragmáticos mejor estudiados desde hace un par de décadas. En una de las primeras aproximaciones sobre la cuestión, R. Lakoff (1973: 295-300) sugirió, por ejemplo, que el intercambio de usted -o su equivalentes en otras lenguas - constituye una de las manifestaciones más claras de la regla de cortesía que la autora norteamericana resume bajo el lema «No te impongas» y que aparece fundamentalmente en aquellos casos en los que junto a la neutralización del factor poder existe un grado mínimo de solidaridad entre los interlocutores ${ }^{8}$.

Más recientemente, Brown y Levinson (1987) han abordado con cierto detalle las relaciones entre las formas de tratamiento y el fenómeno de la cortesía. En su monumental estudio sobre este universal pragmalingüístico, Brown y Levinson parten de la noción psicológica de $f a c e^{9}$ como motor de una serie de estrategias comunicativas destinadas a salvaguardar ciertos deseos e intereses que los interlocutores se otorgan reciprocamente en el curso de la interacción y que pueden adoptar dos semblantes: «the want of every 'competent adult member' that his actions be unimpeded by other (negative face) [or] the want of every member that his wants be desirable to at least some others (positive face)». Desde esta perspectiva, todos los fenómenos, tanto lingüísticos como sociales, relacionados con la cortesía, la deferencia o el tacto presuponen en el fondo la existencia de un potencial de agresividad entre las partes, agresividad que debe ser eliminada para hacer posible la comunicación.

En este contex to de mutua vulnerabilidad, lo lógico es, sin embargo, suponer que cualquier individuo racional tenderá o bien a eliminar aquellos actos que puedan lesionar los «intereses» del interlocutor $-v . g r$. órdenes, amenazas,

8 Otros casos en que se aplica esta misma regla de cortesía en las lenguas occidentales es, por ejemplo, el uso de determinadas preguntas consideradas corteses («May I ask...?»), de oraciones impersonales y pasivas en contextos de formalidad, el empleo del nosotros autorial en los trabajos de investigación o de términos técnicos que enmascaran posibles connotaciones negativas de algunos conceptos. Junto a esta primera regla de cortesía, Lakoff propone otras dos: a) «da opciones» y b) «haz sentirse que tu interlocutor se sienta bien, sé amistoso», a las que hay que añadir una serie de reglas de claridad, que coinciden con las famosas máximas de Girce y que, a juicio de la autra norteamericana, no son más que una subespecie de la regla «No te impongas».

9 Partiendo de la idea goffmaniana (Goffman 1981: 138 y ss.) de la ofensa virtual (virtual offence) que implica el realizar la peor lectura posible de las acciones que un individuo A realiza y que pueden entrar en colisión con los intereses de otro individuo B, Brown y Levinson (1987:61) indican: «our notion of face (...) face up with notions of being embarrased o humiliated, or «losing face». Thus face is something that is emotionally invested, and that can be lost, maintenaided, or enhanced, and must be constantly attended to in interaction. In general, people cooperate (and asume each other's cooperation) in maintaining face in interaction such cooperation being based on the mutual vulnerability of face». 
advertencias, etc... - o bien mitigar sus efectos mediante la adopción de una serie de estrategias comunicativas: «In other words, he will take into consideration the relative weightings of (at least) three wants: a) the want to communicate the content of the FTA $x^{10}, b$ ) the want to be efficient or urgent, and c) the want to maintain H's [hearer] face to any degree. Unless (b) is greater than (c), S [speaker] want to minimize the threat of his FTA» (págs. 68).

El siguiente esquema recoge las macroestrategias que, a juicio de estos autores, son generalmente adoptadas por los hablantes en la interacción verbal. Tales estrategias aparecen jerarquizadas a partir del grado de riesgo que las distintas situaciones comunicativas imponen:

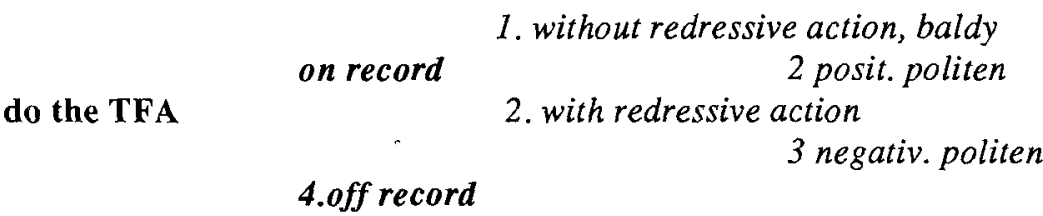

\section{5. don't do the FTA ${ }^{11}$}

(Fuente: Brown, P y Levinson, S (1987: 69))

Por otro lado, y como factores que potencian la elección de estrategias concretas, ocupan un lugar destacado una serie de variables sociológicas que pueden agruparse en tres grupos:

a) la distancia social (D) entre el hablante y el oyente

b) el poder relativo $(\mathrm{P})$ del hablante sobre el oyente

c) el rango $(R)$ de imposición que determinados tipos de actividad lingüística tienen en una comunidad de habla particular.

En todo caso, tales factores no han de ser valorados como parámetros sociológicos objetivos, sino más bien como asunciones psicológicas interiorizadas por los participantes en la interacción.

En opinión de Brown y Levinson (1987: 22), los sistemas de tratamientos y, entre ellos, los representados por alternancias pronominales en lenguas como el español, constituyen una manifestación gramaticalizada de esta clase de estrategias

10 FTA= Face Threatenings Acts.

1 La diferencia entre la elección de estrategias on record y of record estriba en el grado de ambigüedad del acto realizado por el hablante. Mientras en estas últimas se enmascara a través de diversas técnicas ( $v . g r$. la metáfora, la ironía, los actos de habla indirectos..) el verdadero objeto de las intenciones del interlocutor, las estrategias on record dejan vislumbrar tales intenciones desde el principio. A su vez dentro de éstas se distingue entre las estrategias que traducen sus acciones de la forma más clara, directa y concisa posible (1. without redressive action, baldy) ( $v . g r$. cuando la urgencia o la eficiencia comunicativas aconsejan el sacrificio de mayores dosis de tacto), y aquellas otras que defienden en mayor medida la imagen (face) del oyente. Para las diferencias entre la cortesía positiva (positive politeness) y las cortesía negativa (negative politeness), remitimos al último epígrafe de nuestro trabajo. 
destinadas a minimizar los riesgos psicosociales que todo contacto comunicativo comporta: «the kernel idea of our politeness theory, that some acts are intrinsecally threatening to face and thus require 'softening', finds ratification in microcosm in the domain of honorifics». De la valoración de esta idea nos ocuparemos precisamente tras el comentario de una investigación que hemos llevado a cabo recientemente y cuyos datos presentamos a continuación.

\section{Objeto de la investigación.}

\subsection{Hipótesis:}

Tomando como punto de referencia teórico el trabajo de Brown y Levinson, hemos llevado a cabo un estudio empírico sobre el empleo de los pronombres $t u ́$ y usted en una área concreta del español peninsular como la Comunidad Valenciana. El estudio forma parte de un proyecto más amplio con el que pretendemos analizar esta importante esfera de la deíxis social en diversas comunidades de habla españolas, contribuyendo así a la labor emprendida desde hace unas décadas por otros investigadores del mundo hispánico ${ }^{12}$.

El objeto fundamental de la investigación es profundizar en torno a las relaciones entre el uso de las formas pronominales y el fenómeno de la cortesía. Para ello hemos centrado nuestro interés en el análisis de las relaciones conversacionales en las que el principio de distancia social entre los interlocutores es máximo, como corresponde a las situaciones en que dos personas desconocidas entablan un diálogo en el curso de diversas actividades sociales. Siguiendo la categoría situacional del tono del discurso, - «(que) ref ${ }^{\text {ja }}$ a cómo el locutor (hablante o escritor) interactúa con el alocutario (oyenté o lector) en una relación de interlocutores» (Gregory y Carroll 1986: 85)—, podemos caracterizar la situación extralingüística que nos sirve como punto de partida, de la siguiente manera :

a) un grado elevado de formalidad, dado el desconocimiento previo de los participantes entre sí (tono personal de la relación); y

b) una diversidad funcional en las actividades discursivas (tono funcional), diversidad que depende de los objetivos principales perseguidos por los participantes en cada hecho de habla ${ }^{13}$.

12 En la nota 6 de este mismo trabajo puede verse una relación, no exhaustiva, pero sí al menos suficientemente significativa del trabajo realizado. Como ocurre en otros ámbitos de estudio relacionados con la variación geográfica y social, destaca también en el presente caso el mayor esfuerzo desplegado hasta la fecha en las comunidades de habla latinoamericanas.

En la actualidad llevamos a cabo un trabajo de campo similar al aquí descrito en la Comunidad Vasca, región en la que parece estar produciéndose una auténtica «revolución» en los usos pronominales.

13 Factores como el escenario (calle, establecimiento comercial...), las diferencias (generacionales, sexuales, sociales..) entre los participantes, los roles asumidos en determinados contextos por los interlocutores (comprador-vendedor, profesor-alumno...), etc, explican el diferente tono funcional de cada acto discursivo. 
Nos encontramos, en consecuencia, ante situaciones en las, como ya se ha explicado, la racionalidad que se supone a la mayoria de las personas impulsa la aparición de los criterios de deferencia, respeto y tacto en la conversación.

A pesar de las escasas noticias que sobre esta categoría situacional concreta existen para el ámbito del español peninsular, la bibliografía lingüística, así como una cierta experiencia intutitiva previa, parecen sugerir el predominio aplastante de la forma usted en las relaciones conversacionales que acabamos de describir, ya sea en usos asimétricos, por diferencias claras de poder relativo entre los interlocutores ${ }^{14}$, ya en usos simétricos en los que el factor anterior no tiene relevancia. Para Criado de Val (1973: 5), por ejemplo: «En España se trata de tú al interlocutor familiar o de igual o menor categoría. Se trata de ustéd al desconocido o poco conocido, cualquiera que sea su categoría social, y al superior» (el subrayado es nuestro). Por su parte, la rumana Domnita Dumitrescu (1975-76: 83) propone la siguiente distribución de semas para distinguir entre las formas de tratamiento en el español actual:

$\begin{array}{llll}\text { tú } & \text { (- reverencia) } & \text { (+familiaridad) } & \text { (- énfasis) } \\ \text { usted } & \text { (+reverencia }) & \text { (-familiaridad) } & \text { (-énfasis) } \\ \text { el señor } & \text { (+reverencia) } & \text { (-familiaridad) } & \text { (+énfasis) } \\ \text { (su señoría) } & & & \end{array}$

Como puede verse, tanto la reverencia ${ }^{15}$ como la falta de familiaridad entre los participantes en el diálogo parecen imponer como norma la elección de usted.

En fin, Alba de Diego y Sánchez Lobato (1980: 100) presentan el siguiente cuadro como ilustración de las posibilidades de combinación pronominales en el español general :
A) Relaciones simétricas
I. Trato simétrico solidario: túltú.
II. Trato simétrico no solidario: usted/usted
B) Relaciones no simétricas: túlusted_usted/tú.

A partir de estos datos, formulamos la hipótesis de que en el contexto comunicativo elegido, la forma tradicionalmente considerada como forma de respeto o deferencia, usted, será claramente mayoritaria en las elecciones de los hablantes.

\subsection{El trabajo de campo:}

Para la realización del trabajo de campo hemos elegido una muestra de 231 informantes de la ciudad de Valencia. Asimismo y con el objeto de evaluar la posible incidencia en la variación lingüística de ciertos factores sociológicos,

\footnotetext{
14 En tal caso el superior dirigirá tú al inferior.

15 Concepto quizá un poco excesivo para todos los contextos; podría sustituirse con carácter general por «deferencia» o «respeto»
} 
hemos seleccionado las siguientes variables como potencialmente discriminantes:

a) sexo: 116 mujeres

115 hombres

b) edad: Grupo 1 (hasta 25 años): 71

Grupo 2 (25-40 años): 84

Grupo 3 (40-60 años): 54

Grupo 4 (60 años en adelante): 22

c) sexo del destinatario: hombre (35 años aproximadamente)

mujer (35 años aproximadamente) ${ }^{16}$.

d) situación comunicativa: a través de este factor queremos analizar la posible influencia que ejercen ciertos tipos de actividad social en las que los participantes asumen determinados papeles que vienen dados por variaciones en el escenario, la jerarquía social, los objetivos de la comunicación, etc.. Atendiendo a las propias características del trabajo de campo ${ }^{17}$, hemos seleccionado las siguientes categorías situacionales:

1) el bar

2) relaciones alumno-profesor

3) relaciones jerárquicas en el trabajo : inferior/superior

4) la calle ${ }^{18}$

5) vendedor (empleado)/comprador

6) vendedor (jefe)/comprador

7) profesiones liberales (médicos, abogados...)/cliente

A diferencia de otros trabajos anteriores, hemos pre: ${ }^{2}$ rido adoptar una metodología activa para la obtención de los datos lingüisticos, desechando el uso de cuestionarios y tests cuya validez general como instrumentos de investigación empírica no negamos, pero cuya fiabilidad nunca estará a la altura de la que se obtenga directamente del habla espontánea de los informantes (Manes y Wolfson 1981: 116). Consideramos más significativas las muestras tomadas directamente tras la observación sistemática del investigador —especialmente si, como ocurre en este caso, éste es además participante activo en la interacción- que las impresiones subjetivas aportadas por unos informantes cuya valoración puede deformar la realidad en algún grado. Con este objeto hemos llevado a cabo durante algún tiempo un sencillo experimento que consistía ${ }_{16}$ Hay que advertir que la consideración de esta variable se realizó cuando ya una parte del trabajo de campo estaba realizada, lo que ha determinado la existencia de dos muestras de habla un tanto desequilibradas, hecho que, como veremos, se ha dejado notar en la significación estadística del factor. No obstante, nos decidimos finalmente a incluirla tanto por el propio interés de los datos que podía aportar, como sobre todo, para comparar su incidencia con la observada en trabajos anteriores.

17 Véase más abajo la explicación de las principales características del experimento realizado para la obtención de las muestras del habla.

18 Entendiendo por esta variable las relaciones interlocutivas que se producen en cualquier escenario (calle, establecimientos públicos o privados...) y en donde los participantes no adoptan un determinado papel institucional, como por el contrario sí ocurre en las otras categorías situacionales del estudio. 
en anotar -o grabar - cuidadosamente las variantes pronominales que los integrantes de la muestra dirigían a dos interlocutores fijos durante los primeros minutos de un diálogo. Estos eran el propio investigador (35 años) y otra persona (de igual edad) de sexo femenino, incorporada en un momento posterior al comienzo del trabajo de campo con el fin de evaluar la variable ya reseñada del sexo del destinatario.

Añadamos finalmente que los datos han sido traducidos numéricamente en frecuencias absolutas de aparición de cada una de las dos variantes analizadas $(t \tilde{u}$-usted $)$ para su posterior conversión en porcentajes sobre el global. En última instancia, y para averiguar la significación estadística de las diferencias muestrales, hemos realizado un análisis de varianza y una prueba $-t$ (véase apéndice 1)

\subsection{Los datos.}

\subsection{1) Datos generales.}

Como muestra la tabla adjunta, el pronombre usted es más utilizado que la forma $t u ́$ en el contex to de formalidad analizado, dato que resulta coherente con nuestras hipótesis iniciales. Ahora bien las diferencias porcentuales $(57.6 \%$ para $u s t e d ; 42.4 \%$ para $t u ́)$, aun siendo estadísticamente significativas $(t: 2.159$; $p$. 049), resultan menos abultadas de lo que habiamos esperado inicialmente, hecho que parece confirmar la progresión social del pronombre $t u ́$ en muchos ámbitos sociales, incluidos ahora aquellos en los que se da la máxima distancia social por el desconocimiento previo entre los participantes.

\begin{tabular}{lll} 
Mujer & USTED & \multicolumn{1}{c}{ TU } \\
Hombre & $50,90 \%$ & $49,10 \%$ \\
Men 25 & $66,10 \%$ & $33,90 \%$ \\
$25-40$ & $54,90 \%$ & $45,10 \%$ \\
$40-60$ & $41,60 \%$ & $59,40 \%$ \\
Más 60 & $72,20 \%$ & $27,80 \%$ \\
Hombre (d) & $90,90 \%$ & $9,10 \%$ \\
Mujer (d) & $54,90 \%$ & $45,10 \%$ \\
Contx 1 & $65,50 \%$ & $34,50 \%$ \\
Contx 2 & $66,60 \%$ & $33,40 \%$ \\
Contx 3 & $61,10 \%$ & $81 \%$ \\
Contx 4 & $42,50 \%$ & $38,90 \%$ \\
Contx 5 & $65,80 \%$ & $57,50 \%$ \\
Contx 6 & $67,70 \%$ & $34,20 \%$ \\
Contx 7 & $70 \%$ & $32,30 \%$ \\
TOTAL & $57,60 \%$ & $30 \%$ \\
\hline
\end{tabular}

Tabla 1: Distribución de las variantes T/U por grupos sociológicos (\%)

\subsection{2) El sexo.}

Los resultados de la muestra arrojan un comportamiento diferente de ambos sexos como puede apreciarse en el siguiente gráfico (gráfico 1). Mientras los hombres eligen mayoritariamente la forma pronominal de respeto cuando se 
dirigen a un desconocido (66.1\% frente a un $33.9 \%$ para $t \hat{u})$, superando así las medias globales, las mujeres realizan unas elecciones mucho más equilibradas, como prueba el hecho de que el uso de la forma de solidaridad se sitúe casi al mismo nivel que usted (50.9\% para ésta, frente a $49.1 \%$ para $t u ́$ )

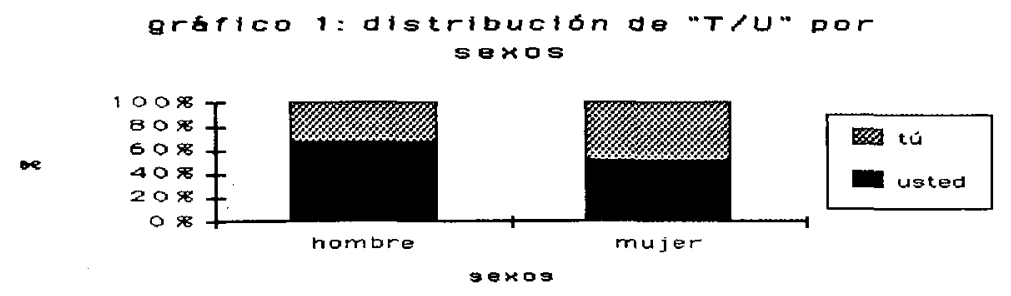

Ahora bien, el análisis estadístico de varianza advierte que no puede descartarse el azar en la configuración de tales diferencias ya que, aun aproximándose a los niveles de significación aceptados comúnmente ( $F .3 .213 ; p .074)$, no están, de hecho, dentro de sus límites. En cualquier caso, estos datos parecen contradecir la hipótesis, tantas veces apı ntada, según la cual el sexo femenino se decanta por las variantes linguiísticas más conservadoras como forma de compensar mediante el prestigio sociolingüístico de sus elecciones la posición desfavorecida que generalmente ocupa en la sociedad (vid Fasold 1990: 92; Silva Corvalán 1989: 70).

La distribución por edades (véase la tabla adjunta) muestra cómo los hombres hacen un mayor uso de la forma de respeto que las mujeres en todos los grupos de edad excepto en el último. A este respecto son especialmente llamativas las diferencias observadas en los grupos $1^{2}$ y $3^{\circ}$ (menores de 25 años y entre 40 y 55 años, respectivamente). En ambos casos -especialmente en el segundo- el porcentaje de empleo de la forma usted es mucho más elevada entre los hombres que entre las mujeres. Tales datos sugieren que la relativa distancia generacional respecto a la edad del interlocutor (recuérdese que los dos interlocutores del experimento pertenecían al grupo 2 de edad) resulta más decisiva entre los miembros masculinos de nuestra comunidad de habla para la elección de una u otra forma pronominal, excepción hecha de los hablantes más adultos (grupo 4 de edad) que masivamente emplean usted sin distinción de sexos. Asimismo, el comportamiento lingüístico de hombres y mujeres se equilibra cuando la proximidad generacional con el interlocutor es máxima (pertenencia al $2^{\circ}$ grupo).

Interesantes parecen también los datos obtenidos tras la combinación de las variables sexo del hablante y sexo del destinatario. Mientras las mujeres muestran un comportamiento bastante homogéneo en su tratamiento hacia ambos interlocutores (con cifras porcentuales próximas en ambos casos a la media global obtenida por su grupo), los hombres se decantan mayoritariamente por el empleo de la forma tradicional de respeto, especialmente - y esto es lo más 
significativo - en el diálogo heterogéneo, es decir, con respresentantes del sexo opuesto.

Por situaciones comunicativas, el hombre muestra un empleo de usted más decidido que la mujer en las relaciones comerciales comprador-vendedor, tanto cuando éste último es un simple empleado, y por consiguiente su posición jerárquica con respecto al cliente es todavía más baja, como cuando es propietario, en cuyo caso las diferencias sociales pueden atenuarse (contextos 5 y 6). Lo mismo ocurre, aunque en menor medida, con las relaciones laborales asimétricas donde el interlocutor ocupa una posición más elevada que el hablante (contexto 3). Por el contrario, en contextos donde la formalidad es máxima, pero las diferencias de poder relativo son menores, o inexistentes (bien sea porque el hablante conoce que la posición social del interlocutor es similar a la suya, bien porque en todo caso aquél es consciente de su estatus elevado, independientemente de la posición del otro participante, como ocurre en el contexto 7), son las mujeres quienes sobresalen por un trato más deferente. En las relaciones alumno-profesor (contexto 2), ambos sexos se decantan claramente por el tuteo, una muestra más de la tendencia que se ha impuesto claramente en nuestro país, como otros trabajos anteriores han ido poniendo de manifiesto (Borrego y otros 1978; Aguado 1980, etc.). Pbr el contrario, el ámbito donde las manifestaciones lingüísticas entre ambos sexos es claramente divergente es el de las relaciones menos formales, aquellas que se producen en situaciones donde un grado todavía elevado de distancia social se ve mitigado por la ausencia de una jerarquización aparente de las relaciones entre los interlocutores. Ello es especialmente evidente en nuestra investigación en el contexto 4, el de las relaciones cotidianas no formales (la calle), en las que frente a una preferencia sin ambages de la mujer por el pronombre $t u ́$, el hombre sigue afirmando el contenido deferente que sugiere la situación comunicativa, mediante el empleo mayoritario de usted.

\begin{tabular}{lllll} 
& Mujer (U) & Mujer (T) & Hombre (U) & Hombre (T) \\
-25 & $19(48,7 \%)$ & $20(51,3 \%)$ & $20(60,6 \%)$ & $13(39,4 \%)$ \\
$26-40$ & $18(39,1 \%)$ & $28(60,9 \%)$ & $17(44,7 \%)$ & $21(55,3 \%)$ \\
$40-60$ & $12(52,1 \%)$ & $11(47,9 \%)$ & $27(87,1 \%)$ & $4(12,9 \%)$ \\
$60-$ & $7(100 \%)$ & $0(0 \%)$ & $13(86,6 \%)$ & $2(13,4 \%)$ \\
Hombre (d) $40(48,7 \%)$ & $42(51,3 \%)$ & $55(60,4 \%)$ & $36(39,6 \%)$ \\
Mujer (d) & $16(50 \%)$ & $16(50 \%)$ & $22(84,6 \%)$ & $4(15,4 \%)$ \\
Contx 1 & $3(50 \%)$ & $3(50 \%)$ & $11(68,7 \%)$ & $5(31,3 \%)$ \\
Contx 2 & $3(25 \%)$ & $9(75 \%)$ & $1(11,1 \%)$ & $8(88,9 \%)$ \\
Contx 3 & $5(55,5 \%)$ & $4(44,5 \%)$ & $6(66,6 \%)$ & $3(33,3 \%)$ \\
Contx 4 & $6(26,1 \%)$ & $17(73,9 \%)$ & $11(64,7 \%)$ & $6(35,3 \%)$ \\
Contx 5 & $25(56,8 \%)$ & $19(43,2 \%)$ & $27(77,1 \%)$ & $8(22,9 \%)$ \\
Contx 6 & $6(54,5 \%)$ & $5(45,5 \%)$ & $15(75 \%)$ & $5(25 \%)$ \\
Contx 7 & $9(81,8 \%)$ & $2(18,2 \%)$ & $5(55,5 \%)$ & $4(44,5 \%)$ \\
\multicolumn{1}{r}{ Total } & $59(50,9 \%)$ & $57(49,1 \%)$ & $76(66,1 \%)$ & $39(33,9 \%)$
\end{tabular}

Tabla 2: Correlación sexo/edad; sexo/destinatario; sexo/situación comunicativa 


\subsection{3) La edad.}

Como cabía esperar, el factor generacional se presenta como decisivo para el análisis de la distribución sociolinguística de las formas de tratamiento. En consonancia con lo mostrado en investigaciones anteriores, las diferencias de edad entre los interlocutores constituyen un factor de primer orden - probablemente el más importante: $F .9 .711 ; p$ : 000 - en la elección de la forma pronominal cuando un hablante se dirige a su audiencia.

$$
\begin{aligned}
& \text { gráfico 2: distribución "T/U" por grupos de } \\
& \text { edad }
\end{aligned}
$$

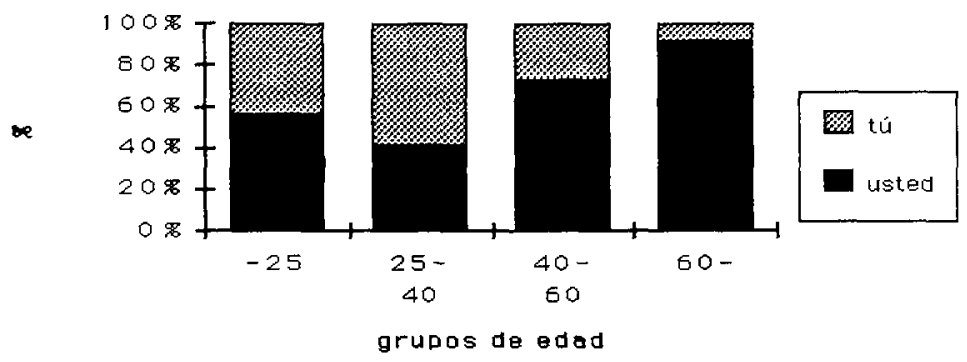

Como muestra el gráfico anterior (gráfico 2) nos encontramos ante una especie de pirámide irregular cuyo vértice reside en el segundo grupo de edad ( 25 a 40 años) y que representa al grupo de informantes claramente favorecedores del tuteo por su proximidad generacional con el interlocutor $(59.4 \%)$. Por otro lado, el calificativo de irregular obedece al hecho de que los dos extremos de la linea no se dibujan de la misma forma en el gráfico. Así, mientras por la derecha, disminuye considerablemente el tuteo, en una progresión descendente que alcanza su punto máximo en el grupo más adulto (mayores de 60), el grupo más joven realiza unas elecciones más equilibradas, mayoritariamente inclinadas todavía hacia la forma de respeto, pero a escasa distancia ya del empleo de $t u ́$.

Estos datos confirman que el avance de la forma pronominal de solidaridad máxima ( $t u$ ) se desarrolla principalmente a partir de los grupos de edad más jóvenes, como otras veces se ha puesto de manifiesto. El claro proceso de cambio lingüistico en el que se hallan inmersas estas formas de tratamiento ha ido fraguando en diferentes situaciones comunicativas, y así tras la conquista por parte de $t u ́$ de diversos ámbitos contextuales en otro tiempo reservados a usted (relaciones familiares, escolares...), la progresión parece que continúa y afecta ya, a la luz de estos datos, a contextos de mayor formalidad. Por el contrario, los hablantes generacionalmente más adultos de nuestra comunidad 
de habla conservan todavía unos usos linguiísticos mucho más acordes con la tradición.

Al margen de la distribución por sexos, para cuya revisión remitimos al epígrafe anterior, hay otros detalles relacionados con el factor edad que merecen ser considerados (véase la Tabla 3). Así, por ejemplo, se aprecia un incremento en el empleo de la forma usted cuando los hablantes de los distintos grupos de edad se dirigen a un interlocutor femenino, mientras que la frecuencia de uso de ese pronombre disminuye en el diálogo con un hombre (salvo el grupo 3). Esta circunstancia cobra una especial significación si comparamos el comportamiento lingüístico del grupo 2, aquél que coincide generacionalmente con el de los destinatarios. Así, mientras en el diálogo con el interlocutor masculino se sigue la norma general - es decir, el empleo relativamente mayoritario de las formas de solidaridad-, cuando el destinatario es una mujer, el esquema se invierte y pasamos a un claro predominio de $u$ sted.

Por lo que se refiere a la distribución por contex tos comunicativos, nuevamente sobresale el comportamiento del grupo 2 , y ello tanto en el uso mayoritario de $t u ́$ en una serie de ámbitos (contextos $1,2,3,4$ ), como por la menor frecuencia relativa de usted en las situaciones más formales (contextos $5,6,7$ ).

$\begin{array}{lllll} & \text { Grup 1(U) } & \text { Grup 1 (T) } & \text { Grup 2 (U) } & \text { Grup 2(T) } \\ \text { Hombre (d) } 31(53,4 \%) & 27(46,6 \%) & 18(32,1 \%) & 38(67,9 \%) \\ \text { Mujer (d) } & 8(61,5 \%) & 5(38,5 \%) & 17(60,7 \%) & 11(39,3 \%) \\ \text { Contx 1 } & 4(80 \%) & 1(20 \%) & 3(30 \%) & 7(70 \%) \\ \text { Contx 2 } & 4(30,7 \%) & 9(69,3 \%) & 0(0 \%) & 6(100 \%) \\ \text { Contx 3 } & 5(100 \%) & 0(0 \%) & 1(14,3 \%) & 6(85,7 \%) \\ \text { Contx 4 } & 2(22,2 \%) & 7(77,8 \%) & 2(18,2 \%) & 9(81,8 \%) \\ \text { Contx 5 } & 23(63,8 \%) & 13(36,2 \%) & 14(53,8 \%) & 12(46,2 \%) \\ \text { Contx 6 } & 0(0 \%) & 2(100 \%) & 8(72,7 \%) & 3(27,3 \%) \\ \text { Contx 7 } & 1(100 \%) & 0(0 \%) & 7(53,8 \%) & 6(46,2 \%) \\ \text { Total } & 39(54,9 \%) & 32(45,1 \%) & 35(41,6 \%) & 49(59,4 \%) \\ & & & & \\ \text { Grup (3) } & \text { Grup 3(T) } & \text { Grup 4(U) } & \text { Grup 4(T) } & \\ 31(73,8 \%) & 11(26,2 \%) & 15(88,2 \%) & 2(11,8 \%) & \\ 8(66,6 \%) & 4(33,3 \%) & 5(100 \%) & 0(0 \%) & \\ 5(100 \%) & 0(0 \%) & 2(100 \%) & 0(0 \%) & \\ 0(0 \%) & 1(100 \%) & 0(0 \%) & 1(100 \%) & \\ 5(83,3 \%) & 1(16,7 \%) & & & \\ 8(53,3 \%) & 7(46,7 \%) & 5(100 \%) & 0(0 \%) & \\ 13(86,6 \%) & 2(13,4 \%) & 2(100 \%) & 0(0 \%) & \\ 5(55,5 \%) & 4(44,5 \%) & 8(88,8 \%) & 1(11,2 \%) & \\ 3(100 \%) & 0(0 \%) & 3(100) & 0(0 \%) & \\ 39(72,2 \%) & 15(27,8 \%) & 20(90,9 \%) & 2(9,1 \%) & \\ & & & & \end{array}$

Tabla 3: Correlación edad/destinatario- edad/situación comunicativa 


\subsection{4) El destinatario.}

Como anunciamos en otro momento, la introducción de esta variable en el estudio se produjo cuando el trabajo de campo se encontraba ya en marcha, por lo que existía el riesgo de que sus resultados globales no tuvieran la validez estadística deseable. $Y$ en efecto, tras el tratamiento informático de los datos obtuvimos unas diferencias entre los dos grupos considerados —diferencias que pueden apreciarse gráficamente en el cuadro adjunto- que no alcanzaban los umbrales de significación aceptables $(F .1 .082 ; p: 299)$, hecho que probablemente haya que imputar en buena parte a esa circunstancia de la propia investigación. No obstante, nuestra experiencia como observadores atentos de la realidad sociolingüística nos impulsó finalmente a valorar la importancia que el sexo del interlocutor podría tener en la elección de las formas de tratamiento. La bibliografía especializada se ha hecho eco en algunos trabajos empíricos de esta misma variable sin obtener unos resultados unánimes ${ }^{19}$.

A la vista de estas circunstancias, los datos que presentamos en esta fase tan sólo pueden ser calificados como orientativos y tienen, por consiguiente, una validez empírica menor que los anteriores. Digamos, no obstante, que al menos en la muestra analizada, se aprecia una leve inclinación a marcar una mayor deferencia hacia el interlocutor femenino, lo que se traduce en un mayor empleo general de usted en tales casos. Esta forma es también mayoritaria en el caso del receptor masculino, aunque a una menor distancia ahora respecto al tuteo (véase gráfico 3).

gráfico 3: distribución "T/U" por sexo del destinatario

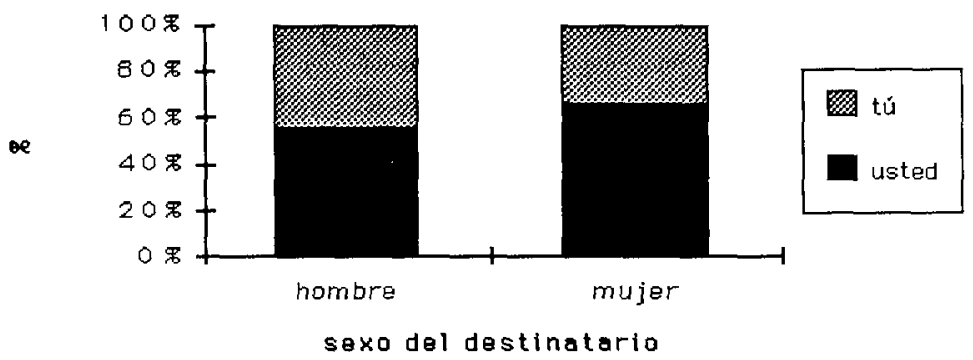

Hombre (U): 95 (54,9\%); Hombre (T): $78(45,1 \%)$

Mujer (U): 38 (65,5\%); Mujer (T): $20(34,5 \%)$

19 Así, parece que se trata de un factor sociológico relevante en el trabajo de Fox (1970:686), pero no en los de Borrego y otros (1978: 62) y Aguado (1980: 180). 
De la distribución de esta variable junto a otras como el sexo o la edad ya se ha hecho alusión en las secciones precedentes, por lo que remitimos directamente a su lectura.

\subsection{5) La situación comunicativa.}

Se trata, sin duda, de la variable más significativa, junto con la edad, de todas las consideradas en nuestra investigación para explicar la variación en los tratamientos pronominales. E1 umbral de significación estadística es de nuevo muy elevado $(F, 3.994 ; p: 001)$ (véase la distribución de frecuencias en el gráfico 4 ).

\section{gráfico 4 : distribución "T/U" por situaciones comunicativas}

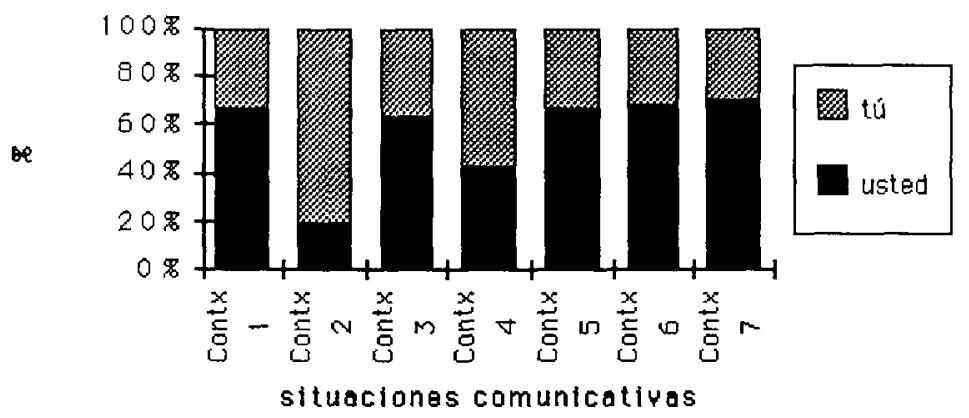

Contx 1 (U): $14(66,6 \%)$; Contx 1 (T): 7 (33,3\%); Contx 2 (U): 4 (19,1\%); Contx 2 (T): $17(80,9 \%)$; Contx 3 (U): $11(62,2 \%)$; Contx 3 (T): $7(37,8 \%)$; Contx 4 (U): 17 (42,5\%); Contx 4 (T): $23(57,5 \%)$; Contx 5 (U): $52(65,8 \%)$; Contx 5 (T): $27(34,2 \%)$; Contx 6 (U): $21(67,7 \%)$; Contx 6 (T): $10(32,3 \%)$; Contx 7 (U): 14 (70\%); Contx 7: 6 (30\%).

La mayoría de los contextos analizados muestran la trascendencia de las diferencias de poder relativo entre los interlocutores cuando alguien se dirige a un desconocido. La mejor prueba de ello es la comparación estadística de las discrepancias entre el contexto 4, donde la capacidad de control sobre el interlocutor es menor, y aquellos contextos - como el 3 o el 5-en los que se observa claramente una cierta jerarquía social entre los participantes en el diálogo. En efecto, es el contexto 4 el único ámbito funcional (a excepción del 
contexto 2, por otras razones que luego comentaremos), en el que se invierte la tendencia general, favoreciendo claramente el tuteo.

En relación con los contextos en que se aprecian unas diferencias de poder relativo entre los interlocutores, cabe recordar que éstas pueden hallarse arraigadas en la realidad social de los individuos, es decir, en diferencias objetivables y evidentes para los participantes en el diálogo, pero también en la asunción psicosocial de papeles sociales e institucionales distintos en determinados escenarios comunicativos (v. gr. relaciones como comprador-vendedor; alumnoprofesor; cliente-camarero, etc...), sin que las discrepancias objetivas en tales ocasiones sean tan obvias. Al primero de los casos mencionados corresponde el contexto 3, que recoge las alocuciones de personal subalterno hacia un superior -en este caso un profesor en el seno de una institución universitaria-. Por el contrario, los contextos 1 (cliente-camarero), 5 (comprador-vendedor asalariado) y 6 (comprador-vendedor propietario) corresponden a la segunda posibilidad. Ahora bien, las diferencias porcentuales entre ambos bloques de grupos son minimas, lo que parece descartar para el presente estudio la relevancia de la distinción efectuada.

Distintos son los resultados que presentan los dos contextos restantes. Por un lado, se ha confirmado plenamente la hipótesis de que ciertas situaciones comunicativas, que tradicionalmente requerían de un tratamiento asimétrico entre los participantes del diálogo, como ocurre en la interacción entre profesor y alumno, han evolucionado en nuestros días de forma considerable. El tuteo predomina con claridad en la alocución del alumno al profesor, lo que coincide plenamente con nuestra intuición de la situación actual. No obstante, habría que matizar esta conclusión indicando que los alumnos de la muestra pertenecían a grupos generacionales próximos al del interlocutor, circunstancia que, sin duda, resulta diferente a aquellas otras en que las diferencias de edad entre ambos participantes son grandes y, por tanto, donde es menos previsible la aparición del tuteo. A este respecto conviene indicar que fueron sobre todo los alumnos de doctorado, más cercanos a la edad del investigador quienes sobresalieron por su tratamiento solidario, a diferencia de los alumnos de los primeros cursos de licenciatura cuyo comportamiento lingiístico fue más variable, alternando el tuteo con el tratamiento de usted .

Por último, la situaciones comunicativas caracterizadas por la relación entre miembros de profesiones liberales (médicos, abogados...) y sus clientes (contex to 7) han respondido plenamente a la norma sociolingüística tradicional basada en el intercambio recíproco de la forma usted. En estos casos, la ausencia del poder como factor decisivo en las relaciones interpersonales se ve compensada tanto por la incidencia de la distancia social máxima que comporta el desconocimiento previo de los interlocutores, como por la clase de actividad social desarrollada, actividades que en nuestra cultura occidental están teñidas 


\section{Reflexiones finales.}

Tanto la bibliografía especializada como la propia experiencia de los hablantes hispanos establecen generalmente para el español moderno un paralelismo entre el uso de usted y diversos factores relacionados con la cortesía, como la deferencia o el respeto, de la misma manera que se relaciona el empleo de la forma $t u ́$ con la familiaridad, la solidaridad o la intimidad entre los interlocutores. Asimismo, es un lugar común que el grado de cortesía aumenta conforme la distancia social entre los participantes en el diálogo es mayor, lo que permite sospechar que en aquellas situaciones sociales marcadas positivamente por esta variable, la elección del llamado pronombre de cortesía, usted, se convierte casi en una regla categórica en el español contemporáneo.

El objeto principal de este trabajo ha sido precisamente comprobar a través de una investigación empírica, en qué medida se cumple esa regla de conducta sociolingüística en una determinada comunidad de habla española. Para ello, se eligió como objeto de análisis un tipo de relaciones humanas en las que el grado de familiaridad entre los interlocutores era mínimo, como corresponde a los primeros momentos de la conversación entre personas desconocidas que se desenvuelven en el seno de diversos tipos de actividad social.

Los resultados de la investigación, sin embargo, permiten rechazar la hipótesis que relaciona categóricamente el empleo de la forma usted en el contexto elegido. Los datos comentados en las secciones anteriores tan sólo apuntan a un uso relativamente mayoritario de este pronombre, pero sin que la elección de la forma tú sea en absoluto desdeñable. Este hecho guarda una relación indudable con la tendencia general observada en otros estudios sobre el español acerca de la ampliación de usos de $t u ́$ en detrimento de usted, en un proceso de cambio continuo cuyas diversas fases todavía hoy pueden constatarse en el habla de diferentes estratos generacionales. Ahora bien, mientras la mayoría de esas investigaciones han comprobado que tal evolución se produce fundamentalmente en el terreno de las relaciones no formales (familiares, amistosas...), los datos obtenidos en nuestro análisis parecen sugerir que el cambio está afectando ya a otros muchos ámbitos sociolingüísticos.

Por otro lado, la sociolingüística demuestra la existencia de una serie de factores no estructurales que aparecen asociados significativamente a la elección de uno u otro pronombre. Por un lado, la edad de los interlocutores se ha revelado como una variable determinante ya que establece unas reglas de comportamiento muy definidas: el empleo de usted aumenta conforme nos alejamos del grupo de edad del receptor, especialmente por la parte alta de la pirámide generacional. En este sentido se aprecia una diferencia importante 
entre las preferencias de los dos primeros grupos de edad, es decir, aquellos que representan a los miembros más jóvenes de la sociedad, y los dos restantes, situados en el otro extremo de la pirámide de edad. La tendencia al uso mayoritario de tú por parte de los primeros contrasta con unos hábitos más tradicionales de las personas más adultas, lo que sin duda es una prueba de los cambios latentes que en torno a este fenómeno sociolingüístico se están produciendo en la sociedad española actual.

Otro factor relevante es el tipo de actividad social desarrollada por los participantes en el intercambio verbal. Así, se ha comprobado que en los contextos donde prima un interés instrumental entre las partes o donde se aprecia claramente una jerarquía social entre ambas, el empleo de usted se contempla todavía como una elección mayoritaria. Por el contrario, otros ámbitos - la calle, el bar, las relaciones alumno-profesor...- determinan un comportamiento sociolingüístico menos conservador .

Por último, cabe recordar - aunque ahora los datos sean menos concluyentesque las mujeres parecen situarse en la vanguardia de los cambios que estamos comentando, con una mayor inclinación que los hombres hacia el tuteo, hecho, por otro lado, que entra en contradicción con el supuesto conservadurismo lingüístico del sexo femenino.

A la luz de estos datos, cabe interrogarse acerca de las relaciones entre el empleo de las formas pronominales de tratamiento y el fenómeno general de la cortesía. ¿Han variado en los últimos tiempos dichas relaciones?, ¿es la sociedad española actual, y particularmente algunos de sus grupos, menos cortés en el trato que hace unas décadas?, ¿cabe pensar, como algunos han supuesto (vid. Alonso 1962), en la futura muerte de usted?. Ciertamente la respuesta a estos interrogantes no es fácil de formular, entre otras cosas porque, como acabamos de ver, parece que nos encontramos en una de las etapas iniciales del cambio descrito, un cambio, por consiguiente, cuyo desenlace está por comprobar. Pero tampoco es ajena a esta dificultad la propia ambigüedad epistemológica que gira en torno al concepto de cortesía.

Recientemente algunos autores han formulado diversas críticas en relación con las formulaciones más comunes en torno a este universal pragmalinguiístico. Para Watts (1992), por ejemplo, el concepto de cortesía manejado últimamente por los lingüistas no coincide con las percepciones que los hablantes de las culturas occidentales suelen tener de ese mismo fenómeno. Estas últimas, que generalmente tienen su origen en las formas de conducta social de las clases altas en distintas cortes europeas desde finales del siglo XVIII (Ehlich 1992: 71 107), discrepan de las estrategias comunicativas ${ }^{20}$ que los hablantes adoptan con

Por cierto, no sólo lingüísticas, sino también paralingüísticas y kinésicas (Brown y Levinson 
el fin de mantener un equilibrio interpersonal en el seno de las relaciones sociales. Por ello, propone un cambio teórico y terminológico en la investigación sobre estos fenómenos: «The various realizations of linguistics politeness in language usage which have been discussed in the literature may more profitably be viewed as forms of a more general form of linguistic behaviour geared towards maintaining the equilibrium of interpersonal relationships within the social group which I have elsewhere termed 'politic verbal behaviour'» (Watts 1992: 43).

Esta reorientación teórica puede ayudar a entender mejor las relaciones entre las formas de tratamiento y ciertas estrategias comunicativas que velan por mantener el equilibrio interpersonal en la interacción. $Y$ en particular pueden aportar una interpretación sociocultural adecuada para la consideración de las formas túlusted del español actual como manifestaciones concretas de las que Brown y Levinson (1987) denominan cortesía positiva y negativa, respectivamente.

En palabras de los propios Brown y Levinson (1987: 101), la llamada cortesía positiva «is redress directed to the addressee's positive face, his perennial desire that his wants (or actions/adquisitions/values resulting from them) should be thought of as desirable». Por consiguiente, se trata de una macroestrategia que recoge todas aquellas técnicas conversacionales destinadas básicamente a relanzar la imagen positiva del receptor.

Uno de los mecanismos más habituales que traducen la realización de esa cortesía positiva consiste en hacer partícipe al interlocutor de una esfera común de intereses, deseos o actividades. Ello da origen a estrategias parciales bien conocidas en la mayoría de las sociedades, como las muestras de un interés exagerado por los intereses del interlocutor, la exaltación de sus habilidades, realizaciones, etc., la búsqueda de motivos de acuerdo en lugar de desacuerdo o el mismo uso de marcas de identidad que subrayan la pertenencia a un mismo ámbito común entre los participantes. En este contexto, el tránsito de las formas pronominales $\mathrm{V}$ a las formas $\mathrm{T}$ en aquellas lenguas que como el español tienen gramaticalizada la alternancia comporta una estrategia de estas características: «In such languages, the use of $\mathrm{T}$ (singular non-honorific pronouns) to a nonfamiliar alter can claim solidarity» (pág 107). Desde esta perspectiva, el uso de tú en el español contemporáneo no sólo abarca el contexto tradicional de las relaciones familiares y amistosas (parentesco, amistad...) sino que, en virtud de su carácter de marcador de proximidad grupal, traspasa su ámbito de uso a otras esferas en las que determinados atributos de los interlocutores pueden inducir a uno de ellos (tratamiento asimétrico) o a ambos (tratamiento simétrico) a su empleo. En consecuencia, la progresión en el empleo de tú en el español de nuestra comunidad sería un reflejo de la tendencia creciente en una sociedad democrática, cada vez más permisiva, a limar prejuicios y jerarquizaciones sociales, determinando una valoración crecientemente positiva del tuteo como 
forma de tratamiento adecuado ${ }^{21}$ en situaciones cada vez más numerosas.

En el extremo contrario, la elección de usted representaría el mantenimiento de estrategias comunicativas más conservadoras y tradicionalmente más prestigiosas que se relacionan con la denominada cortesía negativa: «Negative politeness is redressive action addressed to the addressee's negative face: his want to have his freeedom of action unhindered and his attention unimpeded. It is the heart of respect behaviour, just as positive politeness is the kernel of 'familiar' and 'joking' behaviour (...)When we think of politeness in Western cultures, it is negative-politeness behaviour that springs to mind. In our culture, negative politeness is the most elaborate and the most conventionalized set of linguistic strategies for FTA redress (...) Its linguistic realizations -conventional indirectnesses, hedges on illocutionary force, polite pessimism (about the success of request, etc.), the emphasis on H's relative power- are very familiar and need no introduction» (pág. 129-30).

En particular, el empleo de las formas $\mathrm{V}$ como usted implica la adopción de una estrategia que podríamos resumir bajo el lema «Se deferente» con el receptor y ello tanto si las diferencias entre ambos interlocutores están basadas en el poder (relaciones asimétricas), en la distancia social o el tipo de actividad social desarrollada (relaciones simétricas). Y a la vista de los resultados de nuestra investigación es innegable que esta estrategia sigue siendo mayoritaria en el conjunto de la comunidad analizada, hecho que contradice, al menos por el momento, los negros presagios sobre el futuro del pronombre usted. Factores como la edad adulta de los informantes o determinados escen rios comunicativos instrumentales se revelan decisivos todavía para la consolidación de este comportamiento deferente.

Conclusión: las reglas de alternancia entre $t u ́ u$ y usted en determinados ámbitos comunicativos formales no puede reducirse ya a una elección simplificadora entre comportamientos corteses y no corteses, o si se prefiere, respetuosos v.s. no respetuosos. Más bien constituyen un sistema en el que, cada vez más con más frecuencia, alternan dos tipos diferentes de cortesía - positiva y negativa-. que responden a actitudes y reglas de conducta también diferentes. $Y$ en este sentido, ambas formas pueden ser descritas como claves de contextualización ${ }^{22}$ en el español actual ya que contribuyen a crear entre los participantes del diálogo las presuposiciones necesarias para que infieran determinados significados psicosociales que resultan decisivos para el desarrollo del mismo. La misión de la sociolingüística y de otras disciplinas afines radica, precisamente, en analizar los factores que impulsan a determinados individuos y grupos sociales a la adopción prioritaria de uno u otro modelo en cada comunidad de habla hispánica.

21 Recuérdese, Verbal politic behaviour en la concepción teórica de Watts (1992) con la que básicamente coincidimos.

22 Para la comprensión más detallada de este concepto básico en el desarrollo de disciplinas como la sociolingüística interaccional o la etnografía de la comunicación remitimos al trabajo fundacional de Gumperz (1982). 


\section{Referencias bibliográficas.}

Aguado, D. (1980): «Análisis sociolingüístico del uso de túlusted en los estudiantes universitarios de Bilbao», Letras de Deusto, 21, 1, págs. 165-84.

Alba de Diego, V. y Sánchez Lobato, J. (1980): «Tratamiento y juventud en la lengua hablada. Aspectos sociolingüísticos», Boletín de la Real Academia Española, págs. 95-129.

Alonso, D. (1962): «La muerte del usted», Del siglo de oro a este siglo de siglas, Madrid, Gredos, págs. 264-67.

Borrego, J. y otros (1978): «Sobre el tú y el usted.», Estudia Philologica Salmanticensiae, 3, págs. 53-70.

Brown, D. (1975): «The use of tú and usted with parents by some Mexican American students», Hispania, 58, págs. 126-7.

Brown, P. y Gilman, A. (1960): «The pronouns of power and solidary», en Sebeok, T.A. (ed.), (1960): Style in Language, New York, Wiley, págs. 25376.

Brown, P. y Levinson, S. (1987): Politeness. Some Universals in Language Usage, Cambridge University Press, Cambridge.

Camargo, M. (1972-73): «Formas de tratamento e estructuras sociais», ALfaM, 18/19, págs. 339-81.

Comrie, B. (1976): «Linguistic Politeness Axe: speaker-addressee, speakerreferent, speaker-bystander», Pragmatics Microfiche, 1.7, A:3, Cambridge, University of Cambridge.

Coulmas, F. (1992): « Linguistic etiquette in Japanese Society», en Watts, R y otros (eds.) (1992): Politeness in Language. Studies in its History, Theory and Practice, Berlin, Mouton de Gruyter, págs. 299-324.

Criado de Val, M. (1972): «Los pronombres de cortesía: su tratamiento en español y en otros idiomas. El adverbio», Fisionomía del español y de las lenguas modernas, Madrid, SAETA (también en Yelmo 10, 1973, págs 5-10)

Dumitrescu, D. (1975-76): «Notas comparativas sobre el tratamiento en español y rumano», BSRLR, XI, págs. 81-86.

Eguiluz, M. L. (1962): «Fórmulas de tratamiento en el español de Chile», Boletín de Filología de la Universidad de Chile, XIV, págs. 169-233.

Ehlich, K. (1992): "On the historicity of Politeness» en Watts, R y otros (eds.) (1992): Politeness in Language. Studies in its History, Theory and Practice, Berlin, Mouton de Gruyter, pags. 43-70.

Ervin-Tripp, S. (1972): «On sociolinguistic rules: alternation and co-occurrence» en Gumperz, J y Hymes, D (1972): Directions in Sociolinguistics, New York. Fasold, R. (1990): The sociolinguistics of language, Oxford, Basil Blackwell, Ltd. 
Fillmore, Ch. (1975): «Santa Cruz lectures on deixis», Indiana Univ, Linguistics Club Papers, Bloomington.

Fontanella de Weinberg, M. (1970): «La evolución de los pronombres de tratamiento en el español bonaerense», Thesaurus, XXV, 1, págs. 12-22.

Fontanella de Weinberg, M. y Najt, M. (1969): «Los pronombres de tratamiento en el español de Bahía Blanca», Actas de la Quinta Asamblea Interuniversitaria de Filología y Literatura Hispánica, Universidad Nacional del Sur.

Fox, J. (1970): «The pronouns of adress in Spanish», Actas del X Congreso Internacional de Lingüistas, I, págs. 685-91.

Goffman, E. (1981): Forms of talk, Oxford, Blackwell.

Gold, D (1974): «More on tú and usted», Hispania, 57, 3, págs. 478-9.

Gregory, M. y Carroll, S. (1986): Lenguaje y situación. Variedades del lenguaje y sus contextos sociales., México, Fondo de Cultura Económica.

Gumperz, J. J. (1982): Discourse Strategies, Cambridge, Cambridge University Press.

Keller, G. (1974): «La norma de solidaridad y la de poder en los pronombres de tratamiento. Un bosquejo diacrónico y una investigación del español de Nueva York», The Bilingual Review, 1, págs. 42-58.

Kerbrat-Orecchioni, C. (1990): Les interactions verbales, Paris, Armand Colin. Labov, W., Weinreich, U. y Herzog, M. I. (1968): «Empirical Foundations for

a Theory of Language Change», en Lehmann y Malkiel, Y. (eds.) (1968):Directions for Historical Linguistic: A symposium, University of Texas Press., Austin,, págs. 95-195.

Lakoff, R. (1973): "The Logic of Politeness: or minding your p's and q'a», Papers from the Ninth Regional Meeting of the Chicago Linguistic Society, Chicago Linguistic Society, Chicago, pags. 292-305.

Lapesa, R. (1970): «Personas gramaticales y tratamientos en español», Revista de la Universidad de Madrid, 19, págs. 141-67.

Lastra de Suárez, Y. (1972): «Los pronombres de tratamiento en la ciudad de México», $A L M$, X, págs. 213-17.

Manes, J. y Wolfson, N. (1981): «The compliment formula», en Coulmas, F. (1981): Conversational Routine, The Hague, Mouton.

Marín, D. (1972): «El uso de tú y usted en el español actual», Hispania, 55, págs. 904-08.

Miquel i Vergés, M. (1963): «Fórmulas de tratamiento en la ciudad de México», ALM, III, pảgs. 35-86.

Polo, J. (1975): «El español familiar y formas de tratamiento: ensayo bibliográfico: $7 \gg$, Yelmo, págs. $52-53$.

Schiffrin, D. (1994): Approachs to discourse, Cambridge (Mass), Blackwell. Silva Corvalán, C. (1989): Sociolingüística: teoría y análisis, Madrid, Alhambra. Solé, Y. (1970): «Correlaciones socioculturales del uso del tú,/vos y usted en la Argentina, Perú.y Puerto Rico», Thesaurus, XXV, págs. 161-95. 
Uber, D. R. (1985): «The pronnouns of Address in the Spanish of Bogotá, Colombia», The Secol Review, 8/1, págs. 59-74.

Watts, R. (1992): «Linguistic Politeness and politic behaviour: Reconsidering claims for universality», en Watts, R. y otros (eds.) (1992): Politeness in Language. Studies in its History, Theory and Practice, Berlin, Mouton de Gruyter, págs. 21-42.

Weinerman, C. (1976): Sociolingüística de la forma pronominal, México.

\section{APÉNDICE I.-}

Análisis de Varianza correspondiente a las variables sociológicas de la investigación (Aplicación del Programa Estadístico para Macintosh Systat V. 5.0)

DEP VAR:PRONOMBR N: 231 MULTIPLE R: 0.469 SQUARED MULTIPLE R: 0.220

\section{ANALYSIS OF VARIANCE}

SOURCE SUM-OF-SQUARES DF MEAN-SQUARE F-RATIO P

$\begin{array}{cccccc}\text { SEXO } & 0.646 & 1 & 0.646 & 3.213 & 0.074 \\ \text { EDAD } & 5.854 & 3 & 1.951 & 9.711 & 0.000 \\ \text { DESTINAT } & 0.217 & 1 & 0.217 & 1.082 & 0.299 \\ \text { CONTEXTO } & 4.815 & 6 & 0.803 & 3.994 & 0.001\end{array}$

$\begin{array}{llll}\text { ERROR } & 44.005 & 229 & 0.201\end{array}$

Test de la -t de student para el análisis de las diferencias muestrales entre las variantes T/U (Programa Systat v. 5.0)

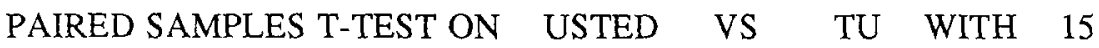
CASES

MEAN DIFFERENCE $=\quad 18.560$

SD DIFFERENCE $=\quad 33.297$

$\mathrm{T}=2.159 \quad \mathrm{DF}=14$

$\mathrm{PROB}=0.049$ 